\title{
A New Online Dynamic Testing Method for Noise of Broadcast Transmitter
}

\author{
Shan-Shan Li $\mathbb{D}$, Jian Zhou, and Xuan Wang \\ Communication University of China, No. 1 Dingfuzhuang East Street, Chaoyang District, Beijing 100024, China \\ Correspondence should be addressed to Shan-Shan Li; 85338101@qq.com
}

Received 20 September 2019; Accepted 20 December 2019; Published 27 February 2020

Academic Editor: Laurie Cuthbert

Copyright (@) 2020 Shan-Shan Li et al. This is an open access article distributed under the Creative Commons Attribution License, which permits unrestricted use, distribution, and reproduction in any medium, provided the original work is properly cited.

\begin{abstract}
Aiming at the shortcomings of traditional broadcast transmitter noise test methods, such as low efficiency, inconvenient data storage, and high requirements for testers, a dynamic online test method for transmitter noise is proposed. The principle of system composition and test method is given. The transmitter noise is real-time changing. The Voice Active Detection (VAD) noise estimation algorithm cannot track the transmitter noise change in real time. This paper proposes a combined noise estimation algorithm for VAD and dynamic estimation. By setting the threshold of the double-threshold VAD detection to be low, it can accurately detect the silent segment. The silent segment is used as a noise signal for noise estimation. For the nonsilent segment detected by the VAD, a minimum value search dynamic spectrum estimation algorithm based on the existence probability of the speech (IMCRA) is used for noise estimation. Transmitter noise is measured by calculating the noise figure (NF). The test method collects the input and output data of the transmitter in real time, which has better accuracy and real-time performance, and the feasibility of the method is verified by experimental simulation.
\end{abstract}

\section{Introduction}

The performance and quality of the broadcast transmitter directly affect the quality of broadcast program. In order to ensure the broadcast quality of the transmitter, it is necessary to carry out regular testing of the transmitter so as to make timely adjustments to the problems that occurred. Noise is one of the main parameters of transmitter performance testing. Traditional static testing methods are manually measured by the tester according to the standard method $[1,2]$. As shown in Figure 1, during the nonbroadcast period of the transmitter, the transmitter is connected to the dummy load, and the audio signal generator generates a single-frequency sine wave signal input transmitter for modulation, the input voltage $U_{s}$ is measured when the amplitude is adjusted to $100 \%$, the output voltage $U_{o}$ is measured when no modulation signal is input, and the SNR is obtained according to the following formula:

$$
\mathrm{SNR}=20 \lg \frac{U_{s}}{U_{o}}
$$

The traditional test method has the following shortcomings [3]. First, it needs to be tested during the nonworking time of the transmitter, and the transmitter status cannot be monitored in real time. If the transmitter fails, it cannot be alerted in time. Second, because the testers have different degrees of mastery of the test instruments and test results will vary at different test times, which results in uncertain test results. Especially when some indicators need to test stability, the tester is required to record the working condition of the device under test for a long time. At this time, the traditional manual method is almost impossible to complete the test requirements. Third, the signal input during the test is a sine wave of a single frequency, but the actual broadcast signal is a multifrequency audio signal.

In view of the shortcomings of traditional measurement methods, this paper presents a new method for online dynamic testing of transmitter noise.

\section{Theoretical Model}

The online dynamic testing system for transmitter noise is shown in Figure 2. 


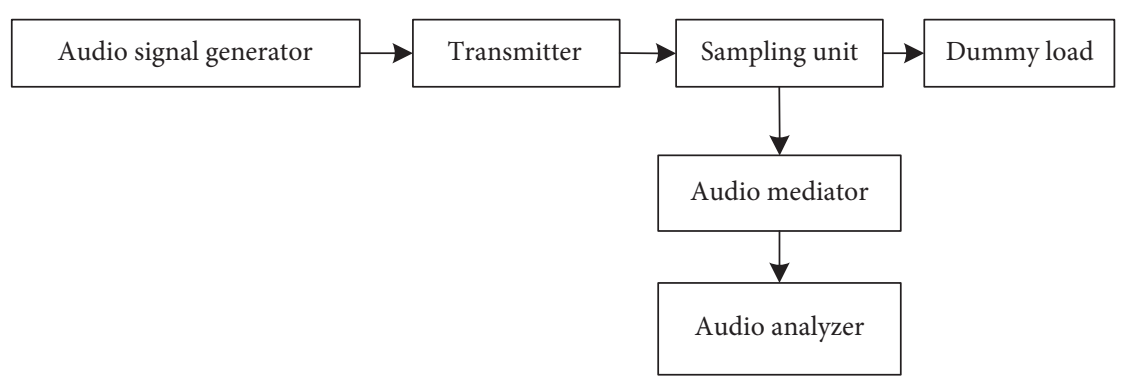

Figure 1: Noise traditional test method.

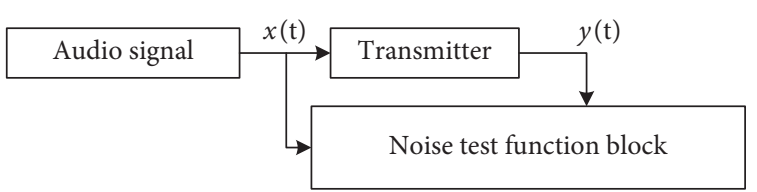

FIGURE 2: Real-time online measurement system model of transmitter parameters.

In Figure 2, an audio broadcast signal $x(t)$ is input to a transmitter, and a signal $y(t)$ is output after being processed by a transmitter. The online test function blocks samples $x(t)$ and $y(t)$ by sampling frequency $f_{s}$ to obtain discretization $x[n]$ and $y[n]$. The signals $x[n]$ and $y[n]$ are processed to obtain the signal-to-noise ratio of the transmitter. The test signal input to the test module is constantly changing over time during the test, so the entire test system structure and test algorithm have changed compared to traditional test methods.

The online test module collects data at regular intervals. The general acquisition interval is $2 \mathrm{~s}$. After the operation processing, it is recollected once, and the data is repeatedly refreshed to realize real-time online testing. The data processing algorithm flow of the noise test function block is shown in Figure 3.

\section{Signal Pretreatment}

In Figure 3, the signal must be preprocessed. For the analog transmitter, the analog broadcast signal is first digitized so that it can be input into the computer for arithmetic processing. Secondly, according to the short-term stationary characteristics of the audio signal, a digital signal input to the computer is windowed and framed, and then each frame signal can be processed. For a digital transmitter, the signal is a digital audio signal, and no digital-to-analog conversion processing is required. Therefore, signal pretreatment plays a very important role in the online test system. Figure 4 shows the main flow of signal pretreatment.

In Figure 4 , the signal $y(t)$ is sampled and quantized to obtain the discrete signal $y[n]$, and then multiplied by $y[n]$ with a window function $w[n]$, and the obtained signal is the framed signal:

$$
\dot{y}[n]=\sum_{n=-\infty}^{\infty} y[k] w[n-k] .
$$

It can be seen from (2) that the method of weighting $y(n)$ by a finite-length movable window function $w[n]$ can realize

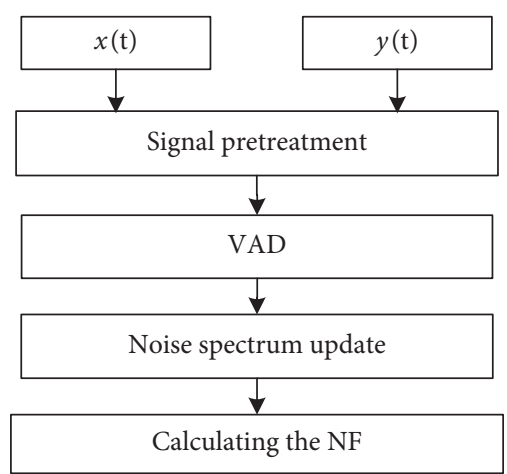

Figure 3: Data processing flowchart of the online test module.

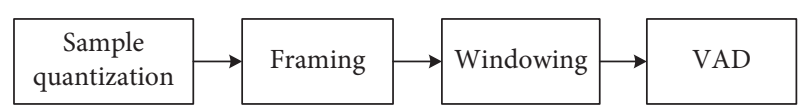

Figure 4: Broadcast signal pretreatment flowchart.

the framing, that is, the broadcast signal after the framing is multiplied by the selected window function $w[n]$ :

$$
y_{w}[n]=w[n] * y(n)
$$

If the signal is multiplied in the time domain, then the convolution operation is performed in the frequency domain, so equation (3) can also be expressed by the following equation:

$$
Y_{w}\left(e^{j w}\right)=\frac{1}{2 \pi} \int_{-\pi}^{\pi} Y\left(e^{j \theta}\right) w\left(e^{j(w-\theta)}\right) \mathrm{d} \theta,
$$

where $w(\cdot)$ and $Y(\cdot)$ represent the window function spectrum and the unwindowed signal spectrum, respectively. It can be seen that the window function $w[n]$ has an effect on the shape of the broadcast signal in the frequency domain, the window function is different, and the resulting windowing function is also different. Rectangular windows, Hanning windows, and Hamming windows are three commonly used window functions.

\section{Broadcast Signal Noise Estimation Algorithm Based on VAD}

The traditional noise estimation algorithm uses VAD to discriminate whether the voice appears and separates the silent segment. At this time, the silent segment mainly exhibits noise characteristics and then obtains some statistical 
methods in the silent region. Update the noise power spectrum with an approximate estimate of the noise characteristics. The threshold-based dual-threshold endpoint detection method assumes that the beginning part of the speech signal is a silent segment without speech, and then the threshold can be set according to the characteristic value of the leading unvoiced segment [4]. The broadcast signal is an uninterrupted continuous audio signal without a leading silence segment, so the conventional method of setting the threshold value as the silence frame for the first few frames of the voice signal is not applicable. If the traditional threshold setting method is used, the threshold will be set incorrectly, eventually causing the endpoint to detect the error. A method for determining the low threshold $T_{\min }$ and the high threshold $T_{\max }$ is given in (5) and (6):

$$
\begin{aligned}
& T_{\text {min }}=\min \left(\max \left(E_{L}, \frac{E_{N}}{4}\right), \frac{E_{N}}{3}\right), \\
& T_{\text {max }}=2.5 * T_{\text {min }},
\end{aligned}
$$

where $E_{N}$ is the short-term energy of the entire audio $y$ :

$$
E_{N}=\sum_{K=1}^{N} y_{i}^{2}(k)
$$

The endpoint detection algorithm is used to continuously estimate and update the average amplitude spectrum of the noise in the input and output signals of the transmitter. The specific steps are as follows:

Step 1: calculate the high and low thresholds of energy from equations (5) and (6), calculate the short-term zero-crossing rate from equation (7), and then use the double-threshold method to determine whether the signal frame is silent.

Step 2: when it is judged as a nonsilent frame, the average amplitude spectrum of the noise is unchanged. When it is judged to be a silence frame, the amplitude spectrum of the noise is updated at this time:

$$
\begin{cases}|N(\lambda, m)|=|N(\lambda, m-1)|, & \text { silence, } \\ |N(\lambda, m)|=\frac{(|N(\lambda, m-1)| * C+|Y(\lambda, m)|)}{C+1}, & \text { no - silence, }\end{cases}
$$

where $N(\lambda, m-1)$ is the average amplitude of the noise before the frame, $N(\lambda, m)$ is the amplitude spectrum of the frame, $c$ is a set constant generally taken as 9 , and equation (8) is to calculate the new one.

\section{Noise Estimation Algorithm Based on Dynamic Spectrum Estimation}

The VAD method is feasible when the noise is stable, but the characteristics of the noise spectrum vary greatly in the actual noise environment $[5,6]$. Under the low SNR, the false detection rate of the VAD will increase, and the silent segment cannot be correctly judged. It is difficult to guarantee the accuracy of the estimated noise. It can also be seen from Figure 4 that the noise spectrum of the VAD algorithm is updated only when the silent segment is detected, and the noise spectrum for the nonsilent segment remains unchanged, and the noise of the transmitter changes in real time. Therefore, in order to achieve accurate transmitter noise estimation, the noise spectrum is estimated in real time [7-9].

In Figure 1, the online test module collects the transmitter signal at regular intervals. The length of the transmitter signal in the time domain is a finite value, and the transmitter output signal is $y(n)$, and the output signal contains a pure audio signal as $s_{y}(n)$ and the noise signal contained in the output signal is represented as $n_{y}(n)$, where $n$ is the sampling time point. Then, the transmitter output signal can be expressed as follows:

$$
y(n)=s_{y}(n)+n_{y}(n) .
$$

Do Fourier transform on both sides of formula (9):

$$
Y\left(\omega_{k}\right)=S_{y}\left(\omega_{k}\right)+N_{y}\left(\omega_{k}\right)
$$

where $\omega \_k=2 \pi k / L, L=0,1,2, \ldots, L-1$, and $L$ is the length of one sample frame, and equation (10) can also be expressed in the polar form:

$$
Y_{k} e^{j \theta_{y}(k)}=S_{y_{k}} e^{j \theta_{s}(k)}+S_{y_{k}} e^{j \theta_{n}(k)},
$$

where $\left\{Y_{k}, S_{y_{k}}, S_{y_{k}}\right\}$ and $\left\{\theta_{y}(k), \theta_{s_{y}}(k), \theta_{s_{y}}(k)\right\}$ represent the pure signal contained in the transmitter output signal and the output signal, respectively, and the amplitude and phase of the noise signal at frequency point $K$. The signal $y(n)$ containing $L$ samples is subjected to frame processing by the window function $w(n)$, and the overlap between frames is set to $R$ point. The FFT operation of the $L$ point for $y(n)$ can transform the noisy signal $y(n)$ into the frequency domain:

$$
Y(\lambda, m)=\sum_{\imath=1}^{L-1} y(\lambda R+\iota) w(\iota) e^{-j 2 \pi \imath m / L}
$$

where the frame number is the time label, $m$ is the label of the frequency component point, $\lambda \in Z, k \in\{0,1, \ldots, \mathrm{L}-1\}$, and $w(l)$ is a window sequence.

Since the speech and noise are assumed to be relatively independent, equation (10) can be approximated as follows:

$$
|Y(\lambda, m)|^{2} \approx|S(\lambda, m)|^{2}+|N(\lambda, m)|^{2},
$$

where $|Y(\lambda, m)|^{2},|S(\lambda, m)|^{2}$, and $|N(\lambda, m)|^{2}$ are, respectively, the noisy signal, the periodogram amplitude of the pure signal, and the noise signal The power spectrum, $\lambda$ represents the frequency and $m$ represents the frame number, that is, the value at the $\lambda$ th frequency of the $m$ th frame. A recursive averaging algorithm based on the probability of existence of a signal is based on two assumptions shown by the following equation:

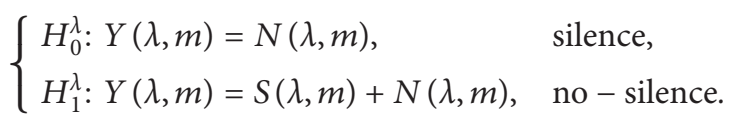


A smoothing process is used to estimate the noise power spectral density $P(\lambda, m)$ :

$$
P(\lambda, m)=\alpha(\lambda, m) P(\lambda-1, m)+(1-\alpha(\lambda, m))|Y(\lambda, m)|^{2} .
$$

Equation (16) gives the calculation of the a optimal value $\alpha_{\text {opt }}$ :

$$
\alpha_{\mathrm{opt}}(\lambda, m)=\frac{\alpha_{\max } \alpha_{c}(m)}{1+\left(p(\lambda-1), m / \sigma_{N}^{2}(\lambda, m)-1\right)^{2}},
$$

where $\alpha_{c}(m)$ can be calculated from the following equation:

$$
\alpha_{c}(m)=\frac{1}{1+\left(\sum_{k=0}^{K-1} p(k, m-1) / \sum_{k=0}^{K-1}|Y(k)|^{2}-1\right)^{2}} \text {. }
$$

The value of $\alpha_{\max }$ in formula (16) is generally 0.96 .

$$
\begin{cases}H_{0}^{\lambda}: \widetilde{\sigma_{N}^{2}}(\lambda, m)=\alpha \cdot \widetilde{\sigma_{N}^{2}}(\lambda, m-1)+1-\alpha|Y(\lambda, m)|^{2}, & \text { silence } \\ H_{1}^{\lambda}: \widetilde{\sigma_{N}^{2}}(\lambda, m)=\alpha \cdot \widetilde{\sigma_{N}^{2}}(\lambda, m-1), & \text { no-silence. }\end{cases}
$$

Equation (18) expresses that the noise power spectral density estimate is constant when the signal is present, and the noise power spectral density estimate is updated when the signal is not present. Equation (19) gives an estimate of the noise power spectral density:

$$
\begin{aligned}
\widetilde{\sigma_{N}^{2}}(\lambda, m)= & E\left[\sigma_{N}^{2}(\lambda, m) \mid Y(\lambda, m)\right] \\
= & E\left[\sigma_{N}^{2}(\lambda, m) \mid H_{0}^{\lambda}\right] p\left(H_{0}^{\lambda} \mid Y(\lambda, m)\right) \\
& +E\left[\sigma_{N}^{2}(\lambda, m) \mid H_{1}^{\lambda}\right] p\left(H_{1}^{\lambda} \mid Y(\lambda, m)\right) .
\end{aligned}
$$

Based on the abovementioned assumptions, $\sigma_{N}^{2}(\lambda, m)$ can be expressed as follows:

$$
\begin{aligned}
\widetilde{\sigma_{N}^{2}}(\lambda, m)= & E\left[\sigma_{N}^{2}(\lambda, m) \mid Y(\lambda, m)\right] \\
= & \alpha \cdot \widetilde{\sigma_{N}^{2}}(\lambda, m-1)+1-\alpha|Y(\lambda, m)|^{2} p \\
& \cdot\left(H_{0}^{\lambda} \mid Y(\lambda, m)\right)+\widetilde{\sigma_{N}^{2}}(\lambda, m-1) p\left(H_{1}^{\lambda} \mid Y(\lambda, m)\right) \\
= & {\left[\alpha \cdot \widetilde{\sigma_{N}^{2}}(\lambda, m-1)+1-\alpha|Y(\lambda, m)|^{2}\right] } \\
& \cdot\left(1-p(\lambda, m)+\widetilde{\sigma_{N}^{2}}(\lambda, m-1) p(\lambda, m)\right),
\end{aligned}
$$

where $p(\lambda, m)=p\left(H_{1}^{\lambda} \mid Y(\lambda, m)\right)$ represents the conditional probability of the presence of a speech signal, and equation (17) can be simplified to

$$
\widetilde{\sigma_{N}^{2}}(\lambda, m)=\alpha_{N}(\lambda, m) \cdot \widetilde{\sigma_{N}^{2}}(\lambda, m-1)+1-\alpha_{N}(\lambda, m)|Y(\lambda, m)|^{2},
$$

where $\alpha_{N}(\lambda, m)$ is obtained by the following formula:

$$
p(\lambda, m)=\frac{1}{1+(q(\lambda, m) / 1-q(\lambda, m))}\left(1+\xi_{\lambda}(m)\right) \exp [-v(\lambda, m)] .
$$

In equation (19), $q(\lambda, m)=p\left(H_{0}^{\lambda}\right)$ is the prior probability of the existence of the speech signal, $v(\lambda, m)=\xi_{\lambda}(m) r_{\lambda}(m) /\left(1+\xi_{\lambda}(m)\right), \xi_{\lambda}(m)$ is the a priori signal-to-noise ratio of the frequency point $\lambda$, and $r_{\lambda}(m)$ is the a posteriori signal-to-noise ratio of the frequency point $\lambda$. $\operatorname{After} p(\lambda, m)$ is obtained by equation (21), it is substituted into equations (15) and (21), and the noise power spectrum can be estimated. A deviation compensation factor of $\beta=1.47$ is also introduced in the noise power spectrum estimation, as shown in the following equation:

$$
\widetilde{\sigma_{N}^{2}}(\lambda, m)=\beta \cdot \widetilde{\sigma_{N}^{2}}(\lambda, m)
$$

According to equation (24), it is necessary to estimate the prior probability $q(\lambda, m)$ in which the speech does not exist, and then the conditional probability $p(\lambda, m)$ can be obtained, and the noisy signal smoothing power spectrum minimum search will be performed. The estimation of $q(\lambda$, $m$ ) has an effect. The IMCRA algorithm performs two smoothing and minimum tracking iterations on the signal. First, a rough voice activity detection is provided for each frequency point by iteration. Then, after an iteration, the relatively strong speech components are eliminated by the smoothing operation, and the shorter window can be used for the minimum search tracking. For highly unstable noise, better tracking results can be achieved with short windows.

In the first iteration, the minimum value tracking search is performed on the past $D$ frame by the estimated value of $S(\lambda, m)$ according to the smoothing formula (15), and $S_{\min }(\lambda, m)$ is obtained by equation (24). To make a rough judgment on the existence of speech,

$$
I(\lambda, m)= \begin{cases}1, \text { 当 } r_{\min }(\lambda, m)<r_{0} \text { 且 } \xi<\xi_{0}, & \text { silence, } \\ 0, & \text { other. }\end{cases}
$$

In equation (24), $r_{0}$ and $\xi_{0}$ are threshold parameters. $r_{\min }(\lambda, m)$ and $\xi(\lambda, m)$ are obtained as shown in formulas (25) and (26), respectively:

$$
\begin{aligned}
r_{\min }(\lambda, m) & =\frac{|Y(\lambda, m)|^{2}}{B_{\min } S_{\min }(\lambda, m)}, \\
\xi(\lambda, m) & =\frac{S(\lambda, m)}{B_{\text {min }} S_{\min }(\lambda, m)},
\end{aligned}
$$


where the $B_{\min }$ factor is the deviation value of the minimum noise estimate. To ensure that the decision to voice is more accurate, $r_{\min }(\lambda, m)$ and $\xi_{\min }(\lambda, m)$ use two different ratios.

In the second iteration, only those frequency components that have been substantially discriminated as noise by equation (24) are smoothed. That is to say, smoothing is mainly for those spectral components containing noise, and $\widetilde{S}_{f}(\lambda, m)$ can be obtained by the following equation:

$$
\widetilde{S}_{f}(\lambda, m)= \begin{cases}\frac{\sum_{i=-L_{w}}^{L_{w}} w(i) I(\lambda-i, m)|Y(\lambda, m)|^{2}}{\sum_{i=-L_{w}}^{L_{w}} w(i) I(\lambda-i, m)}, & \text { if } \sum_{i=-L_{w}}^{L_{w}} I(\lambda-i, m) \neq 0, \\ \widetilde{S}_{f}(\lambda, m-1), & \text { other, }\end{cases}
$$

where $w(i)$ is the window function in the frequency domain and $L_{w}$ is the window length. After calculating the abovementioned $\widetilde{S}_{f}(\lambda, m)$, the first-order smoothing is performed by the following equation:

$$
\widetilde{S}_{f}(\lambda, m)=\alpha_{S} \widetilde{S}(\lambda, m)+\left(1-\alpha_{p}\right) \widetilde{S}_{f}(\lambda, m) .
$$

In the second iteration, a relatively long smoothing window can be chosen because the high energy speech component has been eliminated by the first iteration smoothing. The minimum search window length $D$ can be shortened, which reduces the tracking delay when the noise power increases. The minimum value is tracked on the value of $\widetilde{S}(\lambda, m)$. Let the minimum value of $\widetilde{S}(\lambda, m)$ obtained from equation (28) in the past $D$ frame be $\widetilde{S}_{\min }(\lambda, m)$, and the prior probability $q(\lambda, m)$ of the absence of speech can pass the following formula:

$$
\widetilde{q}(\lambda, m)= \begin{cases}1, & \text { if } \widetilde{r}_{\min }(\lambda, m) \leq 1, \widetilde{\xi}_{\min }(\lambda, m) \leq \xi_{0}, \\ \frac{r_{m}-\widetilde{r}_{\min }(\lambda, m)}{r_{m}-1}, & \text { if } \widetilde{r}_{\min }(\lambda, m) \leq r_{m}, \xi_{\min }(\lambda, m) \leq \xi_{0}, \\ 0, & \text { other, }\end{cases}
$$

where $r_{m}$ is a threshold parameter, $\widetilde{r}_{\text {min }}(\lambda, m)$ and $\widetilde{\xi}_{\min }(\lambda, m)$ are obtained, as shown in formulas (30) and (31), respectively:

$$
\begin{aligned}
\tilde{r}_{\min }(\lambda, m) & =\frac{|Y(\lambda, m)|^{2}}{B_{\min } \widetilde{S}_{\min }(\lambda, m)}, \\
\tilde{\xi}(\lambda, m) & =\frac{\widetilde{S}(\lambda, m)}{B_{\min } \widetilde{S}_{\min }(\lambda, m)} .
\end{aligned}
$$

Equation (29) expresses that when both posterior probability estimates exceed a set threshold, it is indicated that there is speech. When both signal-to-noise ratios based on instantaneous power and smoothed power are below a set threshold, no speech is present. If it is between the two, $q(\lambda, m)$ provides a smooth transition between the presence of speech and the absence of speech. For the weaker speech components, if only $\widetilde{\xi}(\lambda, m)$ is not well judged, $\tilde{\xi}(\lambda, m)$ and $\tilde{r}_{\min }(\lambda, m)$ are used for estimation. During weak speech activity, combined estimation with $\widetilde{\xi}(\lambda, m)$ and $\widetilde{r}_{\min }(\lambda, m)$ can increase the accuracy of noise estimation.

\section{Transmitter Noise Test Algorithm Based on VAD and Dynamic Spectrum Joint Estimation}

When the double threshold setting is low enough, the VAD algorithm will not misjudge the nonsilent segment as a silent segment, but some silent segments will be mistakenly judged as nonsilent segments [10-12]. This paper proposes a VAD and IMCRA joint detection algorithm. The VAD algorithm determines whether the signal is silent. If it is a silent segment, the noise power spectrum is updated by formula (32). If the nonsilent segment is used, the dynamic spectrum update algorithm is used to update the noise power spectrum:

$$
\sigma_{N}^{2}(\lambda, m)=\sigma_{N}^{2}(\lambda, m-1)
$$

where $\sigma_{N}^{2}(\lambda, m)$ and $\sigma_{N}^{2}(\lambda, m-1)$ represent the power spectrum of the current frame noise signal and the power spectrum of the previous frame noise signal, respectively. The average power spectrum of several frames at the beginning of the noisy speech signal is generally used as the initial value of $\sigma_{N}^{2}(\lambda, m)$.

Figure 5 shows the flow of the dynamic update of the broadcast signal noise spectrum.

The flow of the NF online test algorithm is shown in Figure 6 .

The SNR of the transmitter output signal can be obtained by

$$
\mathrm{SNR}_{y}=\frac{S_{0}}{N_{0}},
$$

where $S_{0}$ is the useful signal power of the transmitter output signal, $N_{0}$ is the noise signal power of the transmitter output signal, and $N_{0}$ and $S_{0}$ can be obtained by (34) and (37):

$$
N_{0}=\sum_{\lambda=1}^{D} N_{0}(\lambda)
$$

where 


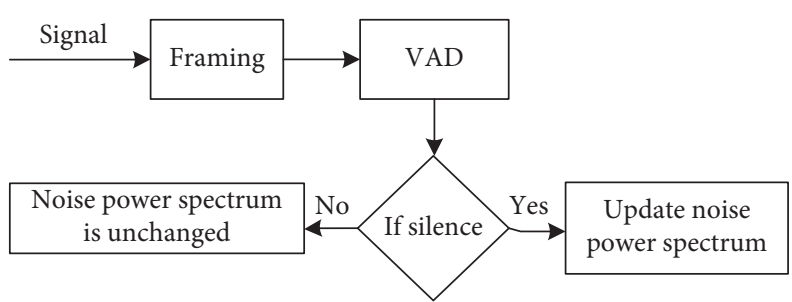

Figure 5: Broadcast signal noise spectrum dynamic update flow chart.

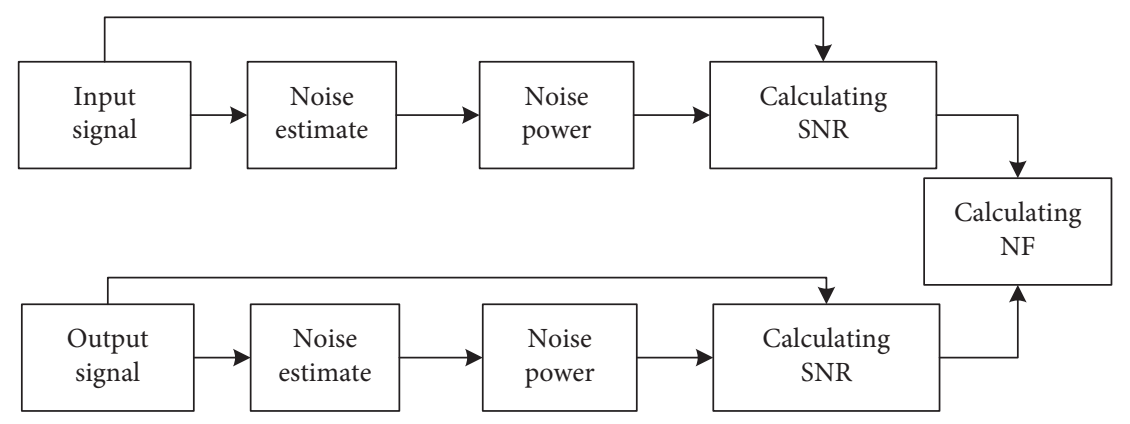

Figure 6: The NF online test algorithm.

$$
\begin{aligned}
N_{0}(\lambda) & =\sum_{m=1}^{L} \sigma_{N}^{2}(\lambda, m), \\
Y_{0} & =\sum_{\lambda=1}^{D} Y_{0}(\lambda),
\end{aligned}
$$

where,

$$
\begin{aligned}
Y_{0}(\lambda) & =\sum_{m=1}^{L}|Y(\lambda, m)|^{2}, \\
S_{0} & =Y_{0}-N_{0} .
\end{aligned}
$$

Similarly, the signal-to-noise ratio of the transmitter input signal $\mathrm{SNR}_{x}$ can be calculated.

The NF of the transmitter is available from the following equation:

$$
\mathrm{NF}=\frac{\mathrm{SNR}_{y}}{\mathrm{SNR}_{x}} .
$$

\section{Results and Analysis}

7.1. Noise Power Spectrum. In order to verify the performance of the noise power spectrum estimation algorithm proposed in this paper, the noise power spectrum estimation algorithm based on IMCRA, the VAD noise power spectrum estimation algorithm, and the proposed noise power spectrum estimation algorithm in this paper are simulated $[13,14]$. Figures 7 and 8 show the effect of the IMCRA algorithm, the VAD algorithm, and the method proposed in this paper on the noise amplitude spectrum when the input signal-to-noise ratio is $30 \mathrm{~dB}$. In order to facilitate the observation and comparison, the true amplitude spectrum of the noise signal is given in each figure.
Figure 7 shows the noise amplitude spectrum estimation at a frequency of $562.5 \mathrm{~Hz}$ for the signal with a signal-tonoise ratio of $30 \mathrm{~dB}$ under the IMCRA and VAD noise estimation algorithms, where the black dotted line is the actual noise amplitude spectrum curve and the black thick curve is the VAD noise estimation algorithm. The resulting noise amplitude spectrum curve, the black fine curve, is the noise amplitude spectrum curve obtained using the IMCRA noise estimation algorithm. It can be seen from the figure that the IMCRA algorithm has better tracking effect on the noise amplitude spectrum than the VAD algorithm. However, in the case of high SNR, the IMCRA algorithm estimates the noise amplitude spectrum around the noise amplitude spectrum without tracking the change in the amplitude spectrum of the noise. The VAD algorithm only tracks the noise amplitude spectrum when the mute portion is detected.

Figure 8 shows the noise amplitude spectrum estimation curve at a frequency of $562.5 \mathrm{~Hz}$ for a signal with a signal-tonoise ratio of $30 \mathrm{~dB}$ under the joint noise estimation algorithm of IMCRA and VAD. The black dotted line is the actual noise spectrum curve, and the black solid line is the estimate noise spectrum by the proposed algorithm in this paper. It can be seen from Figure 7 that the new algorithm uses the IMCRA algorithm to track the noise amplitude spectrum in the nonsilent segment and uses the VAD algorithm to track the noise amplitude spectrum value in the silent segment. The joint estimation algorithm proposed in this paper is better than the IMCRA algorithm or the VAD algorithm. It has a good effect on estimating the noise amplitude spectrum.

7.2. Noise Estimation Performance. In order to test the effect of the noise power spectrum estimation algorithm proposed 


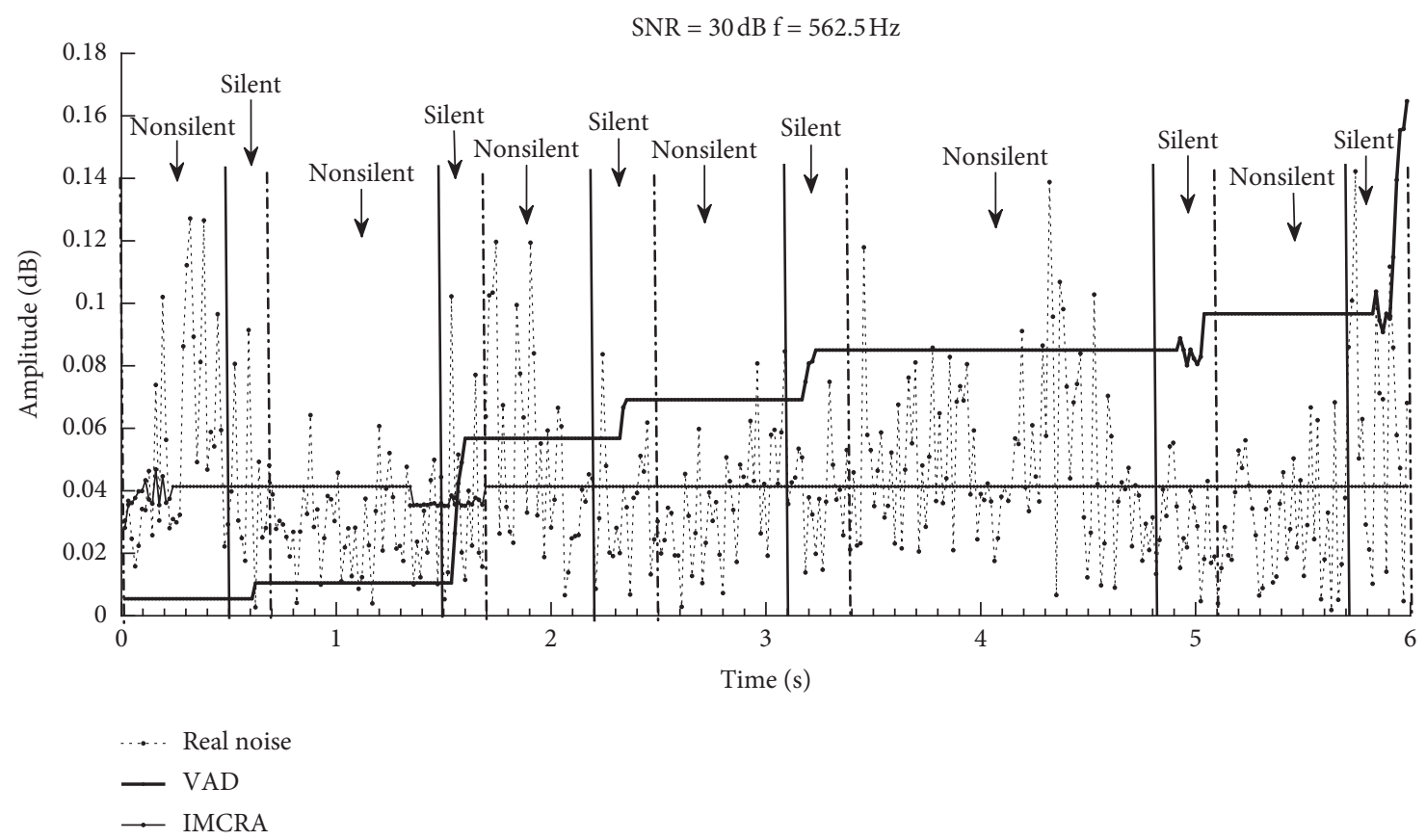

FIGURE 7: The noise amplitude spectrum estimated by VAD and IMCRA.

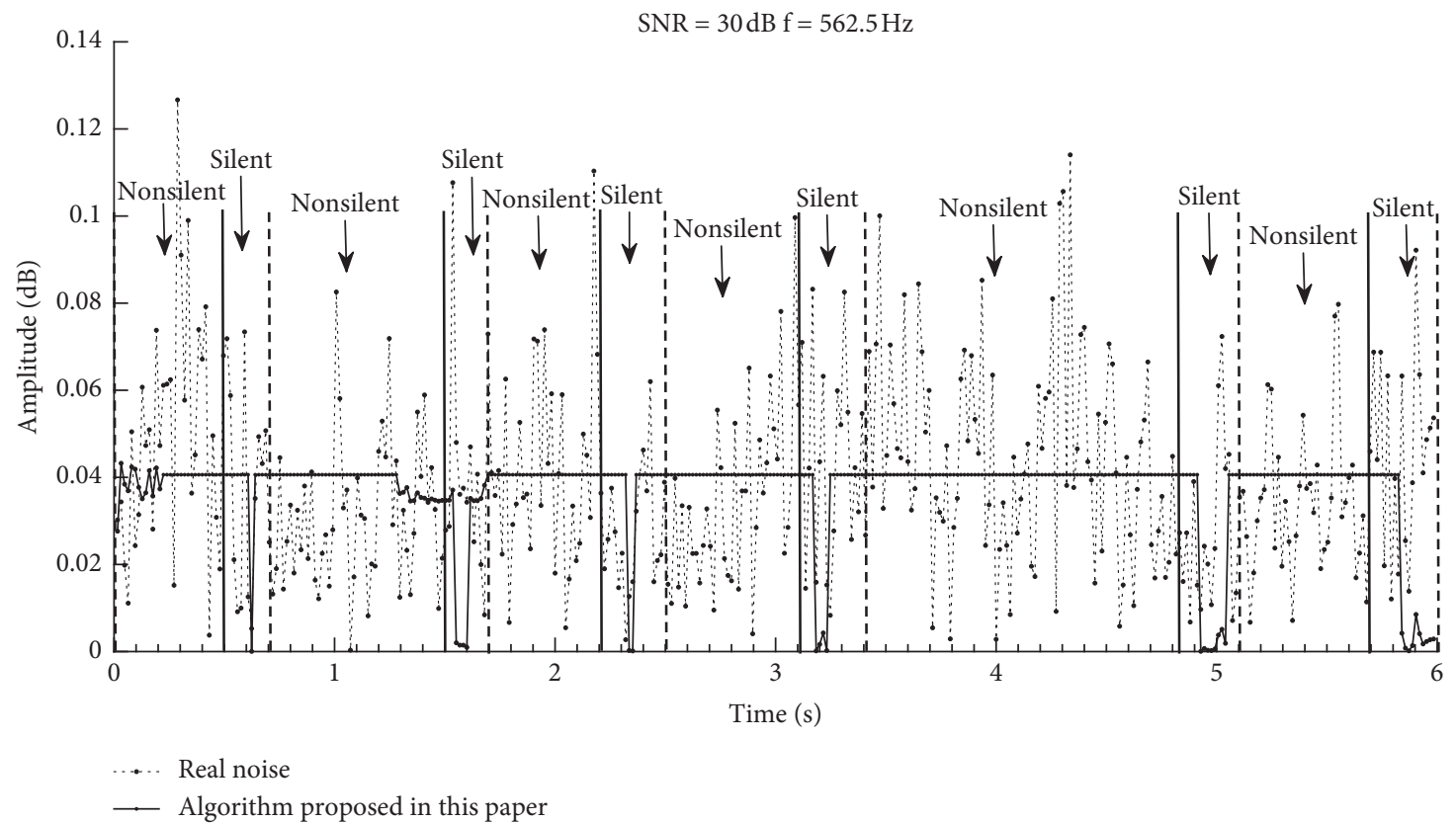

Figure 8: The noise amplitude spectrum estimated by the algorithm proposed in this paper.

in this paper, we compared it with the IMCRA method in this section of the experiment. The comparison includes weighted spectral slope (WSS) and log likelihood ratio (LLR) [15-18]. The noise power spectrum estimation effects of the two algorithms are evaluated. The WSS and LLR simulation results are, respectively, shown in Figures 9 and 10.

In the experiment, a broadcast signal with $6 \mathrm{~s}$ is a useful signal. The noise signal is volvo noise, babble noise, f16 signal Noise, m109 noise, machinegun noise, and white noise from the Noise-92 database. Different noise signals are used as additive noise, and, respectively, mixed with useful signals into noise-containing signals with signal-to-noise ratios of $0 \mathrm{~dB}, 5 \mathrm{~dB}, 10 \mathrm{~dB}, 15 \mathrm{~dB}, 20 \mathrm{~dB}$, and $25 \mathrm{~dB}$, respectively. After the noisy signal is estimated by the noise power spectrum estimation algorithm, the estimated value of the useful signal is obtained by the basic spectral subtraction algorithm, and the WSS is calculated by formula (38) for the estimated signal and the LSS is calculated by formula (39).

The WSS reflects the weighted difference between the spectral slopes at each frequency point, and the difference 

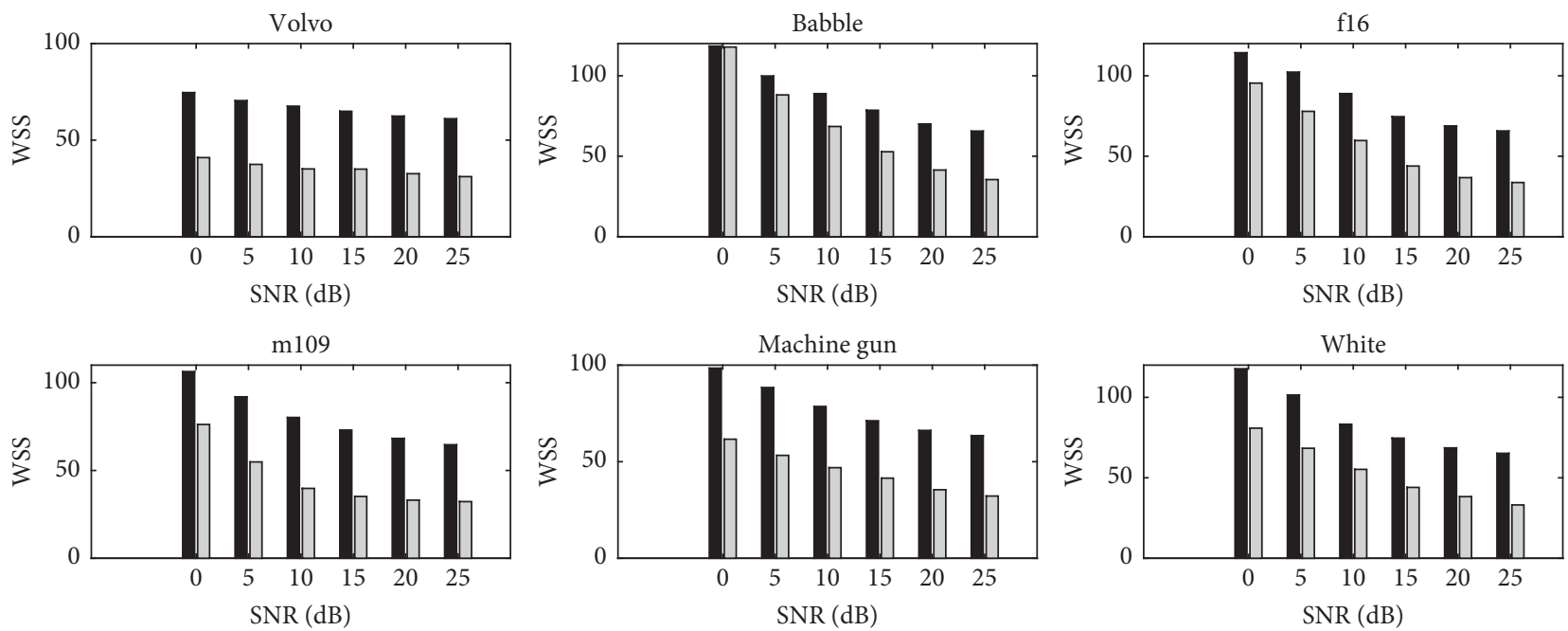

IMCRA

Algorithm proposed in this paper

FIgURE 9: WSS by different noise estimation algorithms. (a) Volvo. (b) Babble. (c) f16. (d) m109. (e) Machinegun. (f) White.
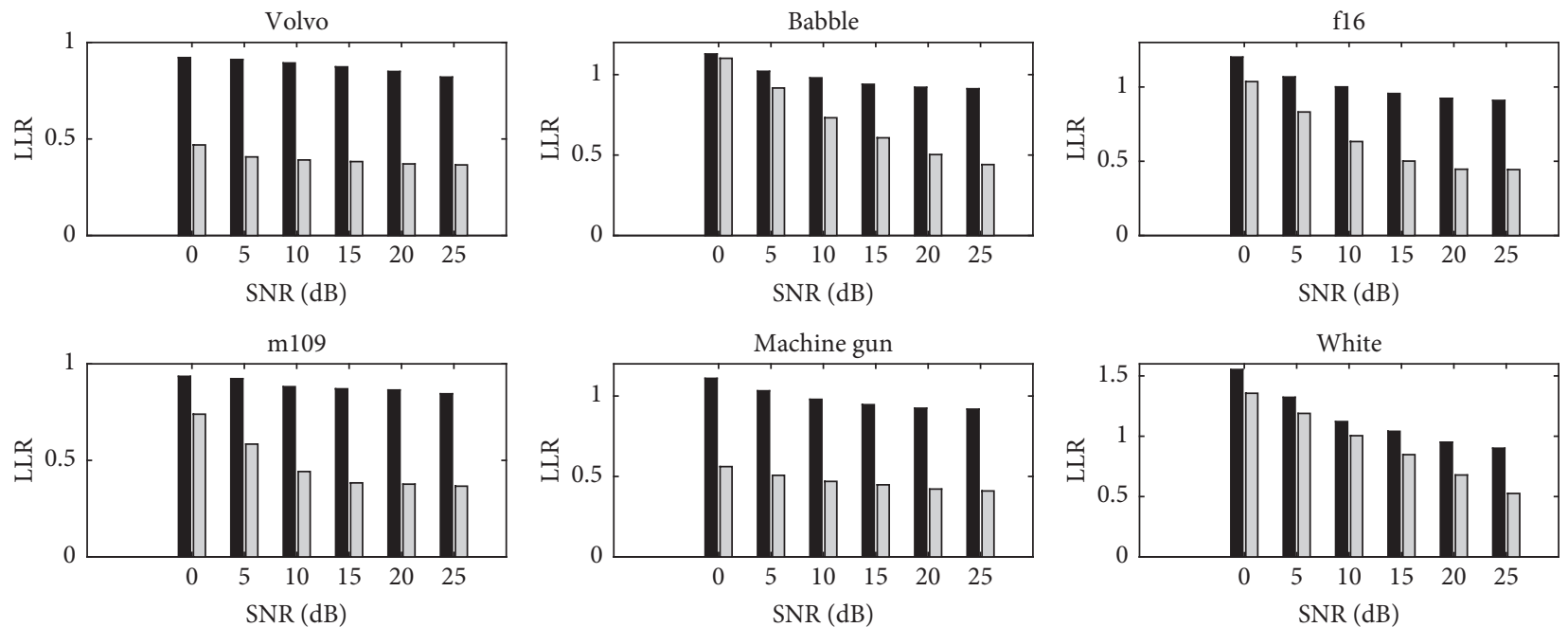

IMCRA

Algorithm proposed in this paper

FIgURE 10: LLR by different noise estimation algorithms. (a) Volvo. (b) Babble. (c) f16. (d) m109. (e) Machinegun. (f) White.

between adjacent amplitude spectra is calculated in the form of decibels. The more smaller the WSS distance, the more closer the estimated signal is to the useful signal, and the better the noise estimation algorithm:

$$
\mathrm{WSS}=\frac{1}{M} \sum_{m=1}^{M} \frac{\sum_{\lambda=1}^{K} W(\lambda, m)(S(\lambda, m)-\widehat{S}(\lambda, m))^{2}}{\sum_{\lambda=1}^{K} W(\lambda, m)},
$$

where $M$ is the total number of frames of the signal, $m$ is the frame index number of the signal, $K$ is the length of the noise frame, and $\lambda$ is the frequency index number, $W(\lambda, m)$ is weighting factor. $S(\lambda, m)$ and $S(\lambda, m)$ are representing the spectral amplitude of the frequency point $\lambda$ of the $m$ frame of the actual useful signal and the estimated useful signal, respectively:

$$
\operatorname{LLR}=\log _{10}\left(\frac{\bar{\alpha}_{\widehat{s}} R_{s} \hat{\alpha}_{\vec{s}}^{T}}{\alpha_{s} R_{s} \alpha_{s}^{T}}\right),
$$

where $\widehat{\alpha}_{\widehat{s}}$ is the linear prediction coefficient vector of the useful signal, $\hat{\alpha}^{T}$ is a linear prediction coefficient vector of the estimated usseful signal, and $R_{s}$ is a linear prediction autocorrelation matrix of the useful signal. The LLR is a spectral distance measure that primarily reflects the degree of mismatch between the estimated useful signal and the original useful signal. The value of LLR ranges from 0 to 2 . The smaller the value is, the smaller the difference between the estimated useful signal and the original useful signal, and the better the performance of the noise estimation is. 


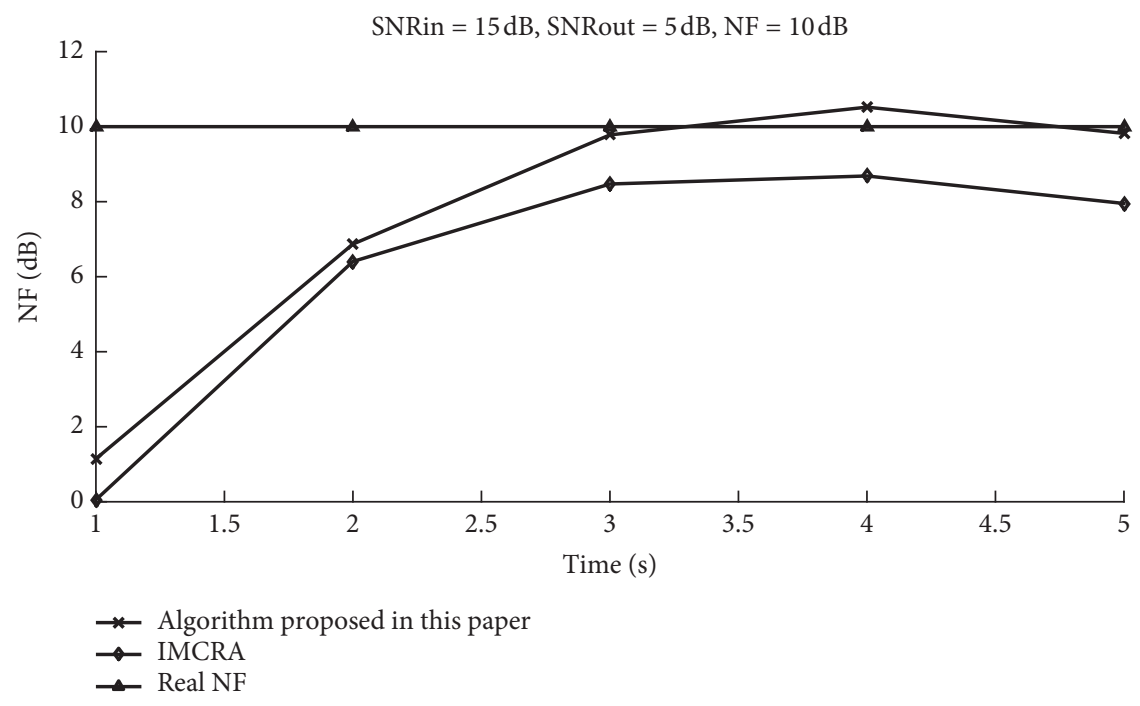

FIGURE 11: NF value curve by different noise estimation algorithms.

By observing the simulation results in Figures 9 and 10, the following conclusions can be drawn:

(1) Under different noise backgrounds and input signalto-noise ratios, the WSS and LLR values of the two noise spectrum estimation algorithms tend to decrease with the increase of the signal-to-noise ratio.

(2) Under different noise backgrounds and input signalto-noise ratios, the proposed IMCRA and VAD joint noise spectrum estimation algorithm has smaller WSS and LLR values than the IMCRA noise spectrum estimation algorithm, indicating that the proposed algorithm is better than the IMCRA algorithm alone. The estimation of the noise spectrum is more accurate.

7.3. Transmitter NF Test Performance. Figure 11 shows the calculated NF value curve after the signal-to-noise ratio of the input signal is $5 \mathrm{~dB}$ and the signal-to-noise ratio of the output signal is $15 \mathrm{~dB}$. The noise is calculated by two different noise estimation algorithms. The square black line is the actual NF value. The diamond black curve is the NF value estimated by $t$ algorithm proposed in this paper, and the triangle black curve is the NF value estimated by the IMCRA algorithm. It can be seen from the figure that the algorithm proposed in this paper is closer to the true value than the NF value estimated by the IMCRA algorithm. After $2.5 \mathrm{~s}$, it fluctuates at nearly $10 \mathrm{~dB}$, which is the fluctuation around the true value, indicating that the proposed method is better for measuring the noise figure of the transmitter.

\section{Conclusion}

The transmitter noise online test algorithm proposed in this paper collects the broadcast audio signal transmitted in real time online, studies the signal-to-noise ratio of the signal to study the noise of the transmitter, and proposes a new dynamic update algorithm for noise spectrum. Compared with traditional test methods, it has better real-time performance. From the simulation results, the new noise spectrum update algorithm proposed in this paper can better track the noise. The error between the NF value and the real value obtained by the online test is very small, which can be used to measure the noise performance of the transmitter. The feasibility of studying transmitter noise performance by measuring the signal-to-noise ratio of broadcast audio signals online is confirmed.

\section{Data Availability}

The data used to support the findings of this study are available from the corresponding author upon request.

\section{Conflicts of Interest}

The authors declare that they have no conflicts of interest.

\section{References}

[1] People's Republic of China Radio, Film and Television Industry Standards, People's Republic of China Radio, China, 2007.

[2] C. Hao and G. Li, "Transmitter parameter measuring methods for digital audio broadcasting in FM band," Radio \& TV Broadcast Engineering, vol. 44, no. 5, pp. 99-102, 2017.

[3] Y. Zhao, TSD-10 DAM Medium Wave Digital AM Broadcast Transmitter Technical Indicators Automatic Comprehensive Test, Newsletter, China, 2018.

[4] D. Y. Yang, W. Zhao, and M. M. Kong, "Broadcast audio segmentation and classification based on endpoint detection," Modern Computer, vol. 11, no. 32, pp. 46-49, 2016.

[5] C. L. Philipos, Speech Enhancement: Theory and Practice, CRC Press, Boca Raton, FL, USA, 2014.

[6] R. Martin, "Spectral subtraction based on minimum statistics," in Proceedings of the 17th EuropeanSignal Processing Conference, vol. 9, no. 5, pp. 1182-1185, Dusseldorf, Germany, November 1994.

[7] I. Cohen and B. Berdugo, "Noise estimation by minima controlled recursive averaging for robust speech 
enhancement," IEEE Signal Processing Letters, vol. 9, no. 1, pp. 12-15, 2002.

[8] I. Cohen, "Noise spectrum estimation in adverse environments: improved minima controlled recursive averaging," IEEE Transactions on Speech and Audio Processing, vol. 11, no. 5, pp. 466-475, 2003.

[9] J. Ma, H. Yu, X. Gong, and X. Zhang, "Research on online measurement method of smartphone energy consumption," in Proceedings of the 2014 Ninth International Conference on P2P, Parallel, Grid, Cloud and Internet Computing, pp. 243247, Guangdong, China, November 2014.

[10] Z. Xu, Q. Ma, L. Liang, and H. Liu, “DVD recorder's audio signals online evaluation method and its measurement system implementation," in Proceedings of the Eighth International Conference on Electronic Measurement and Instruments, pp. 302-306, Xi'an, China, August 2007.

[11] P. J. Púčik, P. Kubinec, and O. Ondráček, "FFT with modified frequency scale for audio signal analysis," in Proceedings of the 2014 24th International Conference Radioelektronika, pp. 1-4, Bratislava, Slovakia, April 2014.

[12] L. Angrisani, M. D’Apuzzo, and M. D’Arco, “New digital signal-processing approach for transmitter measurements in third generation telecommunications systems," IEEE Transactions on Instrumentation and Measurement, vol. 53, no. 3, pp. 622-629, 2004.

[13] V. Natarajan, G. Srinivasan, and A. Chatterjee, "On-line error detection in wireless RF transmitters using real-time streaming data," in Proceedings of the 12th IEEE International On-Line Testing Symposium (IOLTS'06), pp. 1-6, Lake Como, Italy, July 2006.

[14] Y. Hu and P. C. Loizou, "Evaluation of objective quality measures for speech enhancement," IEEE Transactions on Speech and Audio Processing, vol. 16, no. 1, pp. 29-238, 2008.

[15] M. Anouar Ben Messaoud, A. Bouzid, and N. Ellouze, "Speech enhancement based an wavelet packet of an improved principal component analysis," Computer Speech and Language, vol. 35, no. 1, pp. 58-72, 2016.

[16] P. Mowlaee and J. Kulmer, "Harmonic phase estimation in single-channel speech enhancement using phase decomposition and SNR information," IEEE/ACM Transactions on Audio, Speech, and Language Processing, vol. 23, no. 9, pp. 1521-1532, 2015.

[17] M. Shaji Kavalekalam, J. Kjær Nielsen, M. Græsbøll Christensenand, and J. B. Boldt, "A study of noise psd estimators for single channel speech enhancement," in Proceedings of the 2018 IEEE International Conference on Acoustics, Speech and Signal Processing (ICASSP), pp. 5464-5468, Calgary, AB, Canada, April 2018.

[18] Q. Gong, B. Champagne, and P. Kabal, "Noise power spectral density matrix estimation based on modified IMCRA," in Proceedings of the 2014 48th Asilomar Conference on Signals, Systems and Computers, pp. 1389-1395, Pacific Grove, CA, USA, November 2014. 